\title{
MSH2 Protein Variant
}

National Cancer Institute

\section{Source}

National Cancer Institute. MSH2 Protein Variant. NCI Thesaurus. Code C131462.

A variation in the amino acid sequence for DNA mismatch repair protein Msh2. 